\title{
Design a Bioamplifier with High CMRR
}

\author{
Yu-Ming Hsiao, Miin-Shyue Shiau, Kuen-Han Li, Jing-Jhong Hou, Heng-Shou Hsu, \\ Hong-Chong Wu, and Don-Gey Liu
}

Department of Electronic Engineering, Feng Chia University, Taichung 40724, Taiwan

Correspondence should be addressed to Don-Gey Liu; dgliu@fcu.edu.tw

Received 23 December 2012; Accepted 5 April 2013

Academic Editor: Yeong-Lin Lai

Copyright (C) 2013 Yu-Ming Hsiao et al. This is an open access article distributed under the Creative Commons Attribution License, which permits unrestricted use, distribution, and reproduction in any medium, provided the original work is properly cited.

\begin{abstract}
A CMOS amplifier with differential input and output was designed for very high common-mode rejection ratio (CMRR) and low offset. This design was implemented by the $0.35 \mu \mathrm{m}$ CMOS technology provided by TSMC. With three stages of amplification and by balanced self-bias, a voltage gain of $80 \mathrm{~dB}$ with a CMRR of $130 \mathrm{~dB}$ was achieved. The related input offset was as low as $0.6 \mu \mathrm{V}$. In addition, the bias circuits were designed to be less sensitive to the power supply. It was expected that the whole amplifier was then more independent of process variations. This fact was confirmed in this study by simulation. With the simulation results, it is promising to exhibit an amplifier with high performances for biomedical applications.
\end{abstract}

\section{Introduction}

For biomedical applications, a voltage amplifier with a gain of $80 \mathrm{~dB}$ and a high CMRR is required as a building block in front-end subsystems $[1,2]$. Since the voltage level of physiologic signals at the front-end subsystem is very weak, processes for analog signals usually include several steps of amplification, filtering, offset adjustment, and electrical conditioning. After suitable processing, the signal will then be large enough and effectively suitable for analog-to-digital conversion at later stages [3-5].

In considering the physiological signals extracted from human bodies, the amplitude of an electrocardiographic (ECG) signal is usually less than $100 \mu \mathrm{V}$. Such value is very weak as compared to the noise floor and imperfection of the commonly used operational amplifiers (OPAs). An instrumentation amplifier (IA) is usually employed to achieve the required performances.

In addition to the requirement of high voltage gain in constructing the amplifiers for an IA, another important requirement for the amplifiers is CMRR. According to the recommendations of Association of the Advancement of Medical Instrumentation (AAMI), CMRR is required to be higher than $90 \mathrm{~dB}$ with the open-loop voltage gain higher than $80 \mathrm{~dB}$.

In this study, the $0.35 \mu \mathrm{m}$ CMOS technology of TSMC was employed in designing a high performance amplifier.
In our study, a high-voltage-gain amplifier was tried with a self-biasing technique to have a high CMRR and low input offset and to be less sensitive to process variations. The simulation was performed based on the models supported by Chip Implementation Center (CIC). The related results will be illustrated.

\section{Design Details}

2.1. Design of the Differential Amplifier. For the purposes of high CMRR and low offset at the input, differential configuration with a symmetrical floor planning in layout will be preferred in the design of an amplifier.

Figure 1 shows the schematic of an amplifier with the differential configuration both at the input and at the output. In this circuit, transistors $M_{1}$ and $M_{2}$ are the differential pair for amplification. The block with $I_{T}$ and $R_{T}$ forms a tail current bias. The resistors $R_{D 1}, R_{D 2}, R_{L 1}$, and $R_{L 2}$ are taken as the loads.

Figure 2 shows an alternative representation of the amplifier in Figure 1. The input and output signals can be decomposed into the common and the differential modes. With this decomposition, the performance of the amplifier in the common mode and the differential mode can be discussed separately. 


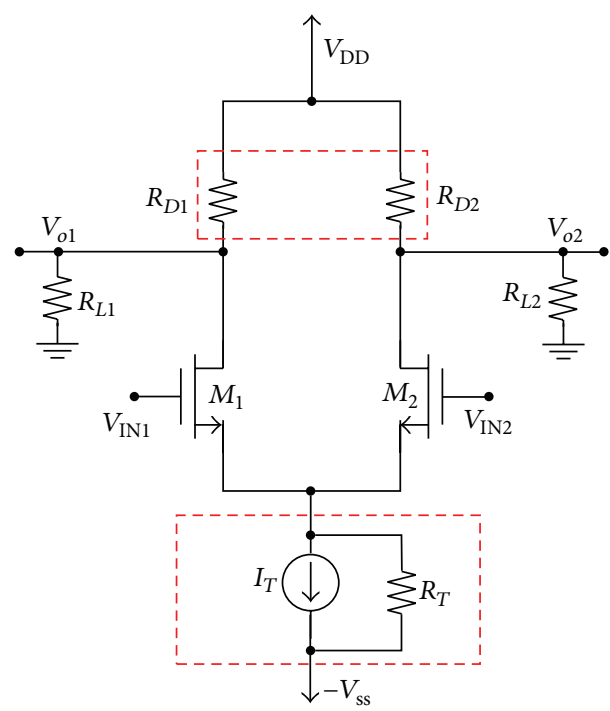

FIGURE 1: Schematic of the differential amplifier.

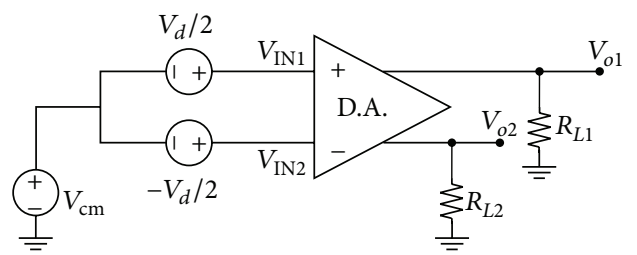

FIGURE 2: Representation of the amplifier in the common and the differential modes.

In the common mode, the two output voltages will be the same if the circuit is ideal in a form of total symmetry. This requires that the branches for $I_{D 1}$ and $I_{D 2}$ are matched with $R_{L 1}=R_{L 2}$ and $R_{D 1}=R_{D 2}$.

For the current bias as the tail, a current mirror with a stable reference current, $I_{\mathrm{REF}}$, can be employed in the integrated circuits to give a high output resistance, $R_{T}$.

Other techniques to improve the performance of this amplifier will be discussed in detail in the following.

For practical design, there exist variations in the devices even with the integrated circuit technology. The output voltage will not be zero for the common input condition. For example, the imperfections in the threshold voltage and the transconductance of the MOS transistors and the variation in $R_{D}$ are uncorrelated. The resulted input offset voltage can be expressed as

$$
\begin{aligned}
V_{\mathrm{OS}} & \equiv \frac{\left.V_{\mathrm{OUT}}\right|_{V_{d}=0}}{A_{d}} \\
& =\frac{V_{\mathrm{OV}}}{2} \sqrt{\left(\frac{\Delta R_{D}}{R_{D}}\right)^{2}+\left(\frac{\Delta(W / L)}{(W / L)}\right)^{2}+\left(\frac{\Delta V_{\mathrm{TH}}}{\left(V_{\mathrm{OV}}\right) / 2}\right)^{2}} .
\end{aligned}
$$

According to the analysis in the common mode and the differential mode, the output voltage can be expressed as the sum of the amplification of the signals of both modes. The relations for the outputs can be written at follows:

$$
\begin{gathered}
V_{\mathrm{O} 1}=\frac{A_{d}}{2} \times v_{d}+A_{\mathrm{cm}} \times v_{\mathrm{cm}}, \\
V_{\mathrm{O} 2}=-\frac{A_{d}}{2} \times v_{d}+A_{\mathrm{cm}} \times v_{\mathrm{cm}},
\end{gathered}
$$

where $v_{d} \equiv v_{\text {in } 1}-v_{\text {in } 2}, v_{\mathrm{cm}} \equiv\left(v_{\mathrm{in} 1}+v_{\mathrm{in} 2}\right) / 2, A_{d}$ is the differential-mode voltage gain, and $A_{\mathrm{cm}}$ is the commonmode gain. It is similar for the expression for $V_{\mathrm{O} 2}$. The common-mode rejection ratio (CMRR) is then defined as

$$
\operatorname{CMRR} \equiv\left|\frac{A_{d}}{A_{\mathrm{cm}}}\right| .
$$

A good amplifier is required to have a high $A_{d}$ with a nearly zero $A_{\mathrm{cm}}$. Due to the variations in the fabrication process, it is a big challenge to achieve a high CMRR with a low input offset. In this study, a balanced bias technique was employed to reduce the sensitivity to the process variation. Good properties of this amplifier have been confirmed in the postlayout simulation.

2.2. Tristage Amplifier. In this design, three stages of amplification were employed to achieve the required voltage gain and CMRR at the same time for weak biosignals. Figure 3 shows the detailed circuit in this design. Table 1 gives the specifications for this design.

As seen in Figure 3, the first stage is composed of $M_{1}-M_{5}$. The second stage includes $M_{6}-M_{12}$. These two stages can be used as an operational transconductance amplifier (OTA) $[6,7]$ or a folded cascade amplifier [8]. The third stage comprising $M_{13}$ and $M_{14}$ forms a type $A$ common-source (CS) amplifier to drive loads.

Transistors $M_{S 1}-M_{S 6}$ provide a bias current for the firststage amplifier. The source of biasing for the second-stage amplifier comes from the balanced self-bias current mirror, $M_{8}-M_{11}$, in Figure 3. In this part, the biasing currents were less sensitive to the level of the power supply. In addition, the complementary arrangement of the loads at the first-stage and the second-stage amplifiers would reduce the variation of the amplification if there are changes in the NMOS and PMOS. The bias voltage for the third stage comes from $M_{9}$ in the second stage. Since the bias currents in $M_{9}$ and $M_{11}$ were constant, the gate bias for $M_{13}$ would be constant. Therefore, the properties of the whole amplifier would be less affected by the uncertainties in fabrication.

For the design strategy, the first stage was designed to achieve a high CMRR rather than a high voltage gain. The overall voltage gain was boosted at the second and the third stages. Since this amplifier was designed for biomedical applications, the voltage gain was tried to be as high as possible with a moderate small bandwidth around $100 \mathrm{~Hz}$. At the third stage, a clamping circuit can keep dynamic tracking of the output gain such that the voltage gain would be less affected by variations in the transistors.

In addition to the electrical considerations, the layout and circuit for the first and second stages were designed as 


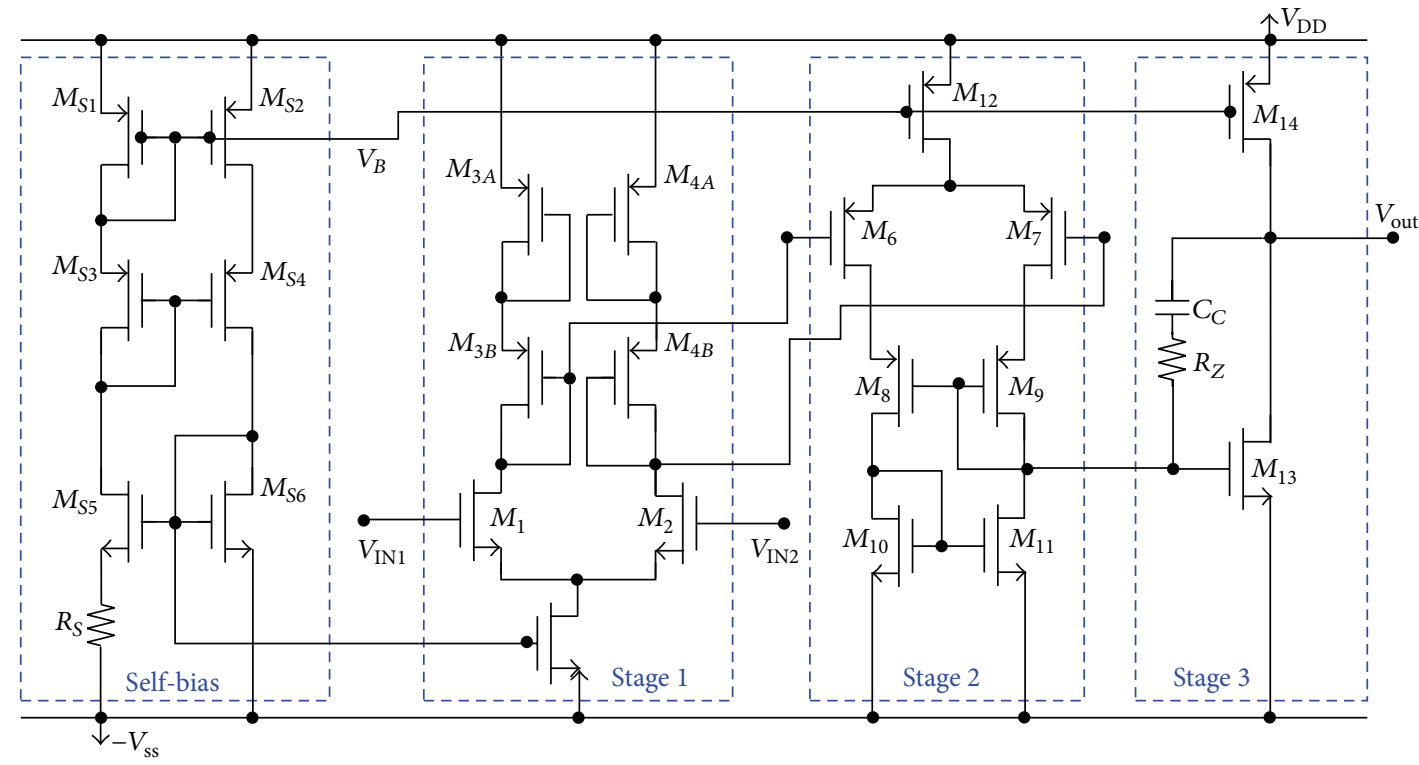

FIgURE 3: Structure of the tristage amplifier.

TABLE 1: Specifications for the bioamplifier.

\begin{tabular}{lcc}
\hline Parameter & Spec. & Value \\
\hline$V_{o} / V_{i}$ & $\geqq$ & $20 \mathrm{kV} / \mathrm{V}$ \\
$A_{\mathrm{VO}}$ & $\geqq$ & $80 \mathrm{~dB}$ \\
$\mathrm{PM}$ & $\geqq$ & $60^{\circ}$ \\
$\mathrm{UGF}$ & $\leqq$ & $2 \mathrm{MHz}$ \\
$\mathrm{CMRR}$ & $\geqq$ & $90 \mathrm{~dB}$ \\
$\mathrm{PD}$ & $\leqq$ & $1 \mathrm{~mW}$ \\
\hline
\end{tabular}

symmetrical as possible. In this way, the common signals would be cancelled out in the differential structure. Therefore, the equivalent input offset would be suppressed effectively.

With the above techniques, an amplifier with high voltage gain, high CMRR, low offset, and low drift voltage can be achieved and confirmed in the simulation.

For our circuit, the level of the power supply was set at $3.3 \mathrm{~V}$ by setting $V_{\mathrm{DD}}$ at $1.65 \mathrm{~V}$ and $-V_{\mathrm{SS}}$ at $-1.65 \mathrm{~V}$. In the meanwhile, the power dissipation was specified below $1 \mathrm{~mW}$ for portable operations. With this constraint, as explained by (4), the total current consumed in this circuit cannot be more than $0.303 \mathrm{~mA}$ :

$$
I_{\text {Supply }} \leq \frac{P_{D, \text { Spec. }}}{V_{\text {Supply }}}=0.303 \mathrm{~mA} .
$$

As for the stability consideration, the phase margin (PM) of this amplifier was tuned to be $60^{\circ}$ in our simulation [9]. In this design, we select $G_{m 3} \geq 10 G_{m 2}$. And the second pole was set as $\omega_{P 2} \geq 2.2 \times \omega_{T}$. Therefore, the Miller compensation capacitor, $C_{C}$, was selected to be $18 \mathrm{pF}$ by the following calculation:

$$
C_{C} \geq 2.2 \times \frac{G_{m 1}}{G_{m 2}} \times C_{\mathrm{O} 2}=0.22 C_{\mathrm{O} 2} .
$$

2.3. Transistor Dimensions. In general, the tail current was required to be higher than the product of the screw rate and $C_{C}$. In this study, this product was $10 \mu \mathrm{A}$ for the second stage. Since the screw rate for the case of light loads is not required strictly, the tail current at stage 2 was selected as $15 \mu \mathrm{A}$.

The bias currents for the two branches through $M_{6}$ and $M_{7}$ equally divide the tail current into $I_{D 6}=I_{D 8}=I_{D 10}=$ $I_{D 7}=I_{D 9}=I_{D 11}=7.5 \mu \mathrm{A}$.

With this bias current, the transconductance, $g_{m}$, and the gate-to-source voltage, $V_{\mathrm{GS}}$, can be designed by a suitable dimension ratio, $W / L$, by the following relations:

$$
\begin{aligned}
I_{D}= & \frac{1}{2} \cdot \mu_{n} \cdot C_{o x} \cdot\left(\frac{W}{L}\right) \cdot\left(V_{\mathrm{OV}}\right)^{2} \cdot\left(1+\lambda V_{\mathrm{DS}}\right), \\
g_{m} & =\left(\mu_{n} \cdot C_{o x}\right) \cdot\left(\frac{W}{L}\right) \cdot\left(V_{\mathrm{OV}}\right) \cdot\left(1+\lambda V_{\mathrm{DS}}\right) \\
& =\sqrt{2 \mu_{n} \cdot C_{o x} \cdot\left(\frac{W}{L}\right) \cdot I_{D} \cdot\left(1+\lambda V_{\mathrm{DS}}\right)} \\
& =\frac{2 \times I_{D}}{V_{\mathrm{OV}}},
\end{aligned}
$$

where the overdrive voltage $V_{\mathrm{OV}} \equiv V_{\mathrm{GS}}-V_{\mathrm{th}}$.

For the third stage, its transconductance gain, $G_{m 3}$, is the same as $g_{m 13}$ of $M_{13}$. We chose $G_{m 3} \geq 10 G_{m 2}$; that is, $G_{m 3} \geq$ $590 \mu \mathrm{A} / \mathrm{V}$ and $g_{m 13}=G_{m 3}=600 \mu \mathrm{A} / \mathrm{V}$. In addition, the overdrive voltage for $M_{13}$ was selected as $V_{\mathrm{OV} 13}=0.2 \mathrm{~V}$; that is, $V_{\mathrm{GS} 13} \equiv V_{\mathrm{OV} 13}+V_{\mathrm{tn}}=0.85 \mathrm{~V}$. Therefore, $V_{\mathrm{DS} 13}=V_{\mathrm{DS} 14}=$ $1.65 \mathrm{~V}$. The dimension ratio for $(W / L)_{13}$ can be determined by (6).

In this design, we used $M_{S 1} \sim M_{S 6}$ and a resistor $R_{S}$ to form a self-bias circuit with a boot-strapping positive 
TABLE 2: Variations of the simulated properties of the bio-amplifier with 5 fabrication corners.

\begin{tabular}{|c|c|c|c|c|c|}
\hline Items & SS & SF & TT & FS & $\mathrm{FF}$ \\
\hline$V_{o} / V_{i}$ & $-33.1 \mathrm{k}$ & $-29.7 \mathrm{k}$ & $-29.3 \mathrm{k}$ & $-27.7 \mathrm{k}$ & $-22.6 \mathrm{k}$ \\
\hline$A_{\mathrm{VO}}(\mathrm{dB})$ & 90.4 & 89.5 & 89.3 & 88.9 & 87.1 \\
\hline $\mathrm{PM}$ & $88.8^{\circ}$ & $89.8^{\circ}$ & $89.7^{\circ}$ & $89.7^{\circ}$ & $91.0^{\circ}$ \\
\hline$f_{\mathrm{H} 3 \mathrm{db}}(\mathrm{Hz})$ & 63.0 & 63.1 & 79.4 & 63.1 & 82 \\
\hline UGF (Hz) & $1.41 E+06$ & $1.47 E+06$ & $1.46 E+06$ & $1.44 E+06$ & $1.52 E+06$ \\
\hline CMRR (dB) & 136.4351 & 136.2238 & 136.7342 & 136.5733 & 135.778 \\
\hline $\mathrm{PD}(\mu \mathrm{W})$ & 357 & 332 & 328 & 324 & 316 \\
\hline$V_{\mathrm{os}}(\mu \mathrm{V})$ & 10.8 & 10.1 & 5.2 & 0.033 & 20.8 \\
\hline
\end{tabular}

feedback. The current controlled by $I_{\mathrm{REF}}$ can be expressed as

$$
I_{\mathrm{REF}} \times R_{S}=V_{\mathrm{GS}_{\mathrm{S} 6}}-V_{\mathrm{GS}_{S 5}}=\sqrt{\frac{I_{\mathrm{REF}}}{K_{S 6}}}-\sqrt{\frac{I_{\mathrm{REF}}}{K_{S 5}}} .
$$

By (8), we selected the dimension ratio of $M_{S 5}$ to be $1 / 4$ of that of $M_{\mathrm{S} 6}$, that is,

$$
\left(\frac{W}{L}\right)_{S 5}=\frac{1}{4}\left(\frac{W}{L}\right)_{S 6} .
$$

The resistance can be obtained as follows:

$$
R_{S}=\frac{1}{I_{\mathrm{REF}}} \sqrt{\frac{I_{\mathrm{REF}}}{K_{S 5}}} .
$$

The reference current was set as $I_{\mathrm{REF}}=5 \mu \mathrm{A}$. The dimension ratio for $M_{S 1} \sim M_{S 4}$ can also be derived by (6).

\section{Simulation and Verification before Fabrication}

In this study, HSPICE with the device models for $0.35 \mu \mathrm{m}$ CMOS technology from TSMC was employed for the simulation and analysis. The performance of the whole circuit was verified first in the prelayout simulation. Then, the physical layout was implemented and the related parameters were extracted. With the obtained information of the physical layout, the postlayout simulation was performed to check the feasibility of our layout. Corner simulations were also performed to check the effect of the process variation on the performance of our amplifier.

Figures 4-7 illustrate the related performances with 5 corner conditions in fabrication. Table 2 lists other performance items with the 5 corners. With these results, we can find that the voltage gain in Figure 6 can be kept higher than $80 \mathrm{~dB}$. And the variation of the obtained gains due to the uncertainty in fabrication can be smaller than $5 \mathrm{~dB}$. It can also be found in Figures 4 and 5 that the phase margin is much larger than $60^{\circ}$. In Figure 5, we can confirm that the variation of phases is insignificant. As shown in Figure 7, the obtained CMRR is as high as $130 \mathrm{~dB}$ for frequency up to $10 \mathrm{kHz}$. The variation of CMRR due to the fabrication is also insignificant. With these results, a bio-amplifier both with a very high CMRR and a high voltage gain at the same time can be expected for the fabricated chips.

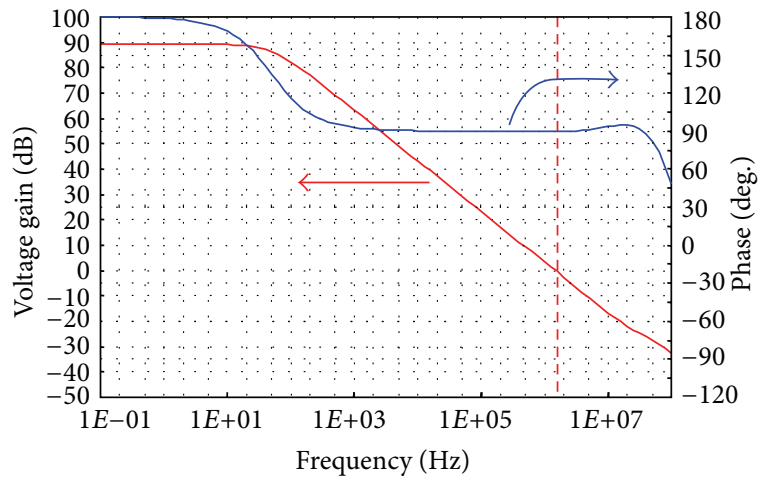

FIGURE 4: Frequency response for the bio-amplifier in the typical fabrication condition (TT).

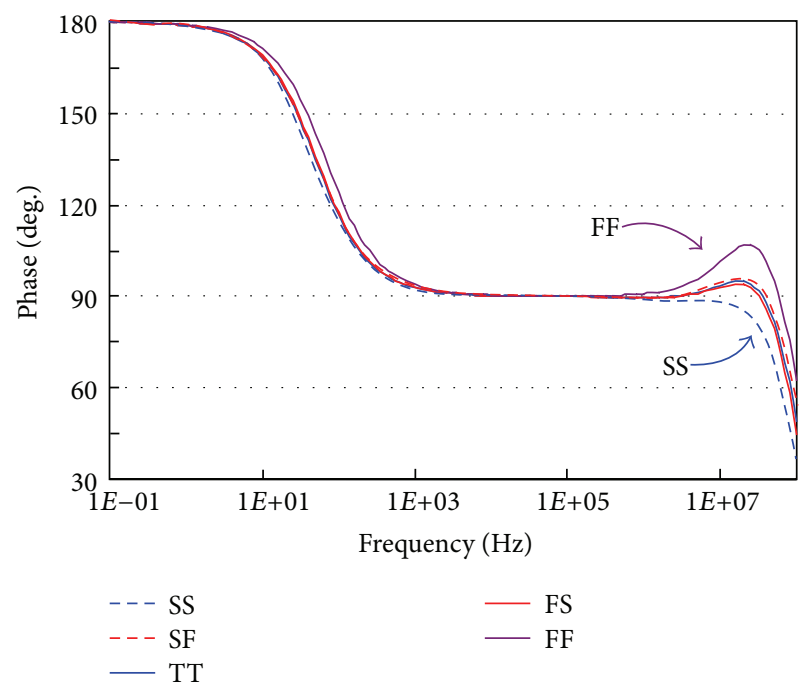

FIgURE 5: Simulated phases for the bio-amplifier in 5 corner conditions.

\section{Conclusion}

A bio-amplifier with high gain and high CMRR was designed and verified in this study. According to the obtained performance properties in Table 2 , it is promising that a process independent performance can be obtained for this amplifier. 


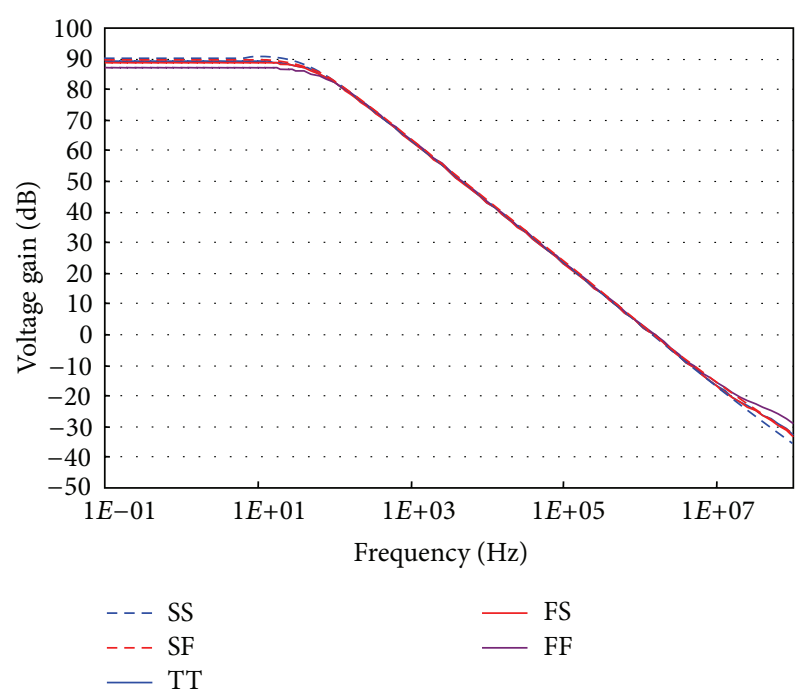

FIGURE 6: Simulated gains for the bio-amplifier in 5 corner conditions.

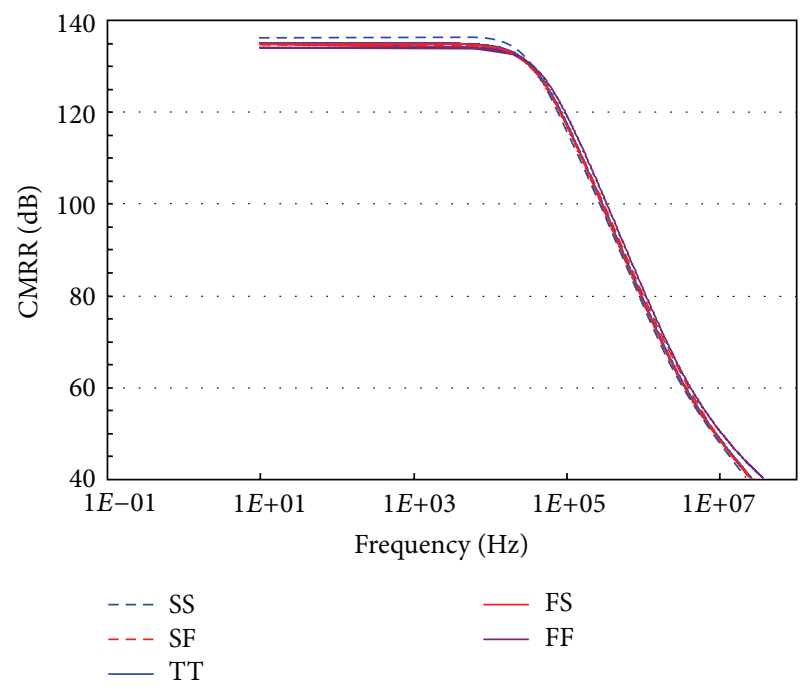

Figure 7: Simulated CMRRs for the bio-amplifier in 5 corner conditions.

\section{Acknowledgments}

The authors acknowledge the support from Chip Implementation Center (CIC) and Chang Bin Show Chwan Memorial Hospital with the research resources. Partial financial support from National Science Council (NSC), republic of china, is also acknowledged.

\section{References}

[1] K. A. Ng and P. K. Chan, "A CMOS analog front-end IC for portable EEG/ECG monitoring applications," IEEE Transactions on Circuits and Systems I, vol. 52, no. 11, pp. 2335-2347, 2005.

[2] B. Wang, H. Ji, Z. Huang, and H. Li, "A high-speed data acquisition system for ECT based on the differential sampling method," IEEE Sensors Journal, vol. 5, no. 2, pp. 308-311, 2005.

[3] C. H. Chan, J. Wills, J. LaCoss, J. J. Granacki, and J. Choma Jr., "A novel variable-gain micro-power band-pass auto-zeroing CMOS amplifier," in Proceedings of the IEEE International Symposium on Circuits and Systems (ISCAS '07), pp. 337-340, May 2007.

[4] B. Premanode, N. Silawan, and C. Toumazou, "Drift reduction in ion-sensitive FETs using correlated double sampling," Electronics Letters, vol. 43, no. 16, pp. 857-859, 2007.

[5] J. Wu, G. K. Fedder, and L. R. Carley, "A low-noise low-offset chopper-stabilized capacitive-readout amplifier for CMOS MEMS accelerometers," in Proceedings of the IEEE International Solid-State Circuits Conference (ISSCC '02), pp. 428-425, February 2002.

[6] G. Nicollini and C. Guardiani, "3.3-V 800-nV rms noise, gainprogrammable CMOS microphone preamplifier design using yield modeling technique," IEEE Journal of Solid-State Circuits, vol. 28, no. 8, pp. 915-921, 1993.

[7] V. Ivanov, J. Zhou, and I. M. Filanovsky, "A 100-dB CMRR CMOS operational amplifier with single-supply capability," IEEE Transactions on Circuits and Systems II, vol. 54, no. 5, pp. 397-401, 2007.

[8] P. C. de Jong, G. C. M. Meijer, and A. H. M. van Roermund, "A $300^{\circ} \mathrm{C}$ dynamic-feedback instrumentation amplifier," IEEE Journal of Solid-State Circuits, vol. 33, no. 12, pp. 1999-2008, 1998.

[9] K. N. Leung and P. K. T. Mok, "Analysis of multistage amplifierfrequency compensation," IEEE Transactions on Circuits and Systems I, vol. 48, no. 9, pp. 1041-1056, 2001. 

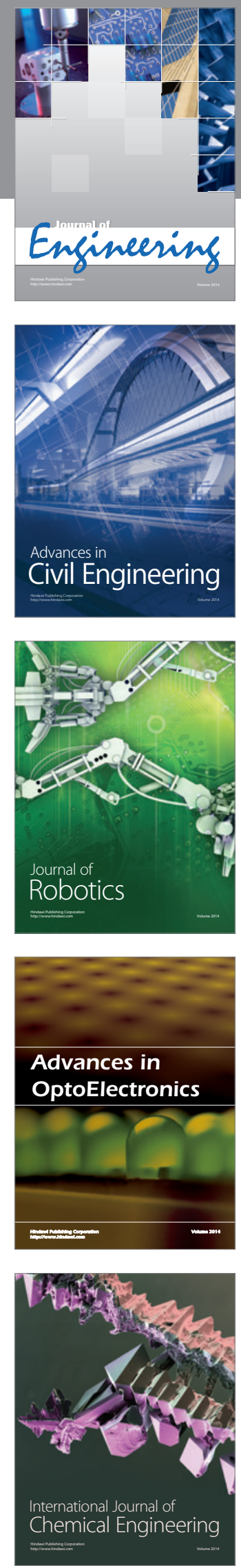

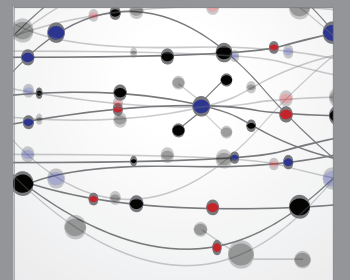

The Scientific World Journal
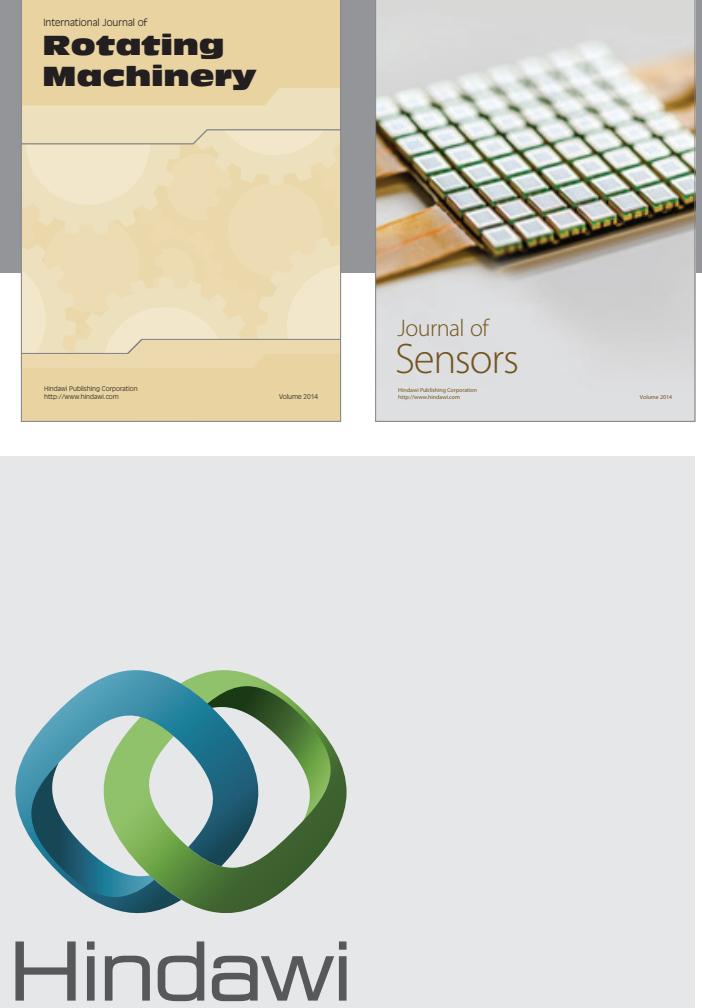

Submit your manuscripts at http://www.hindawi.com
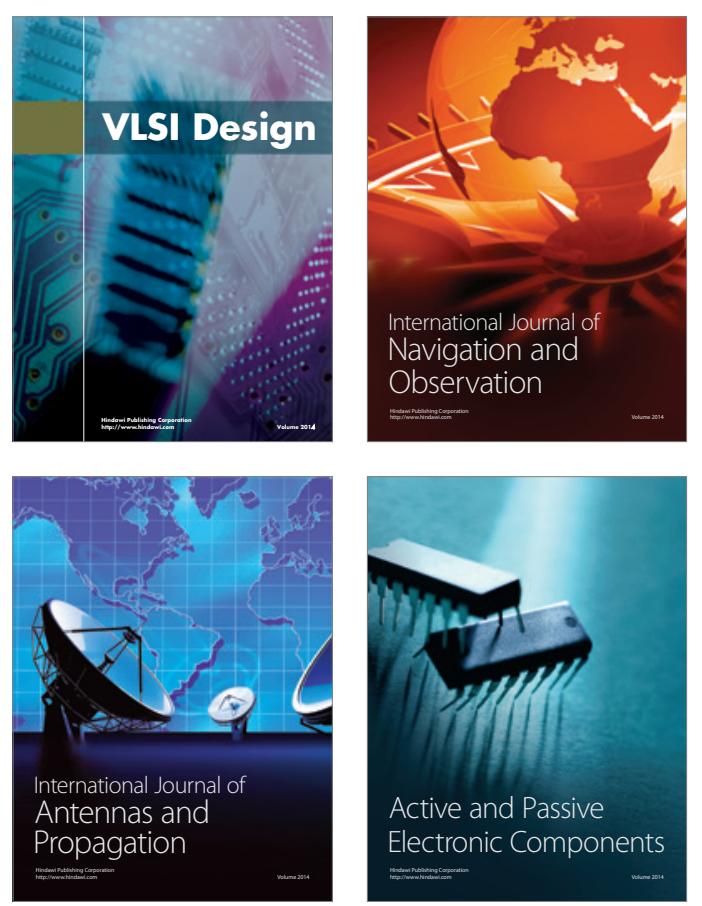
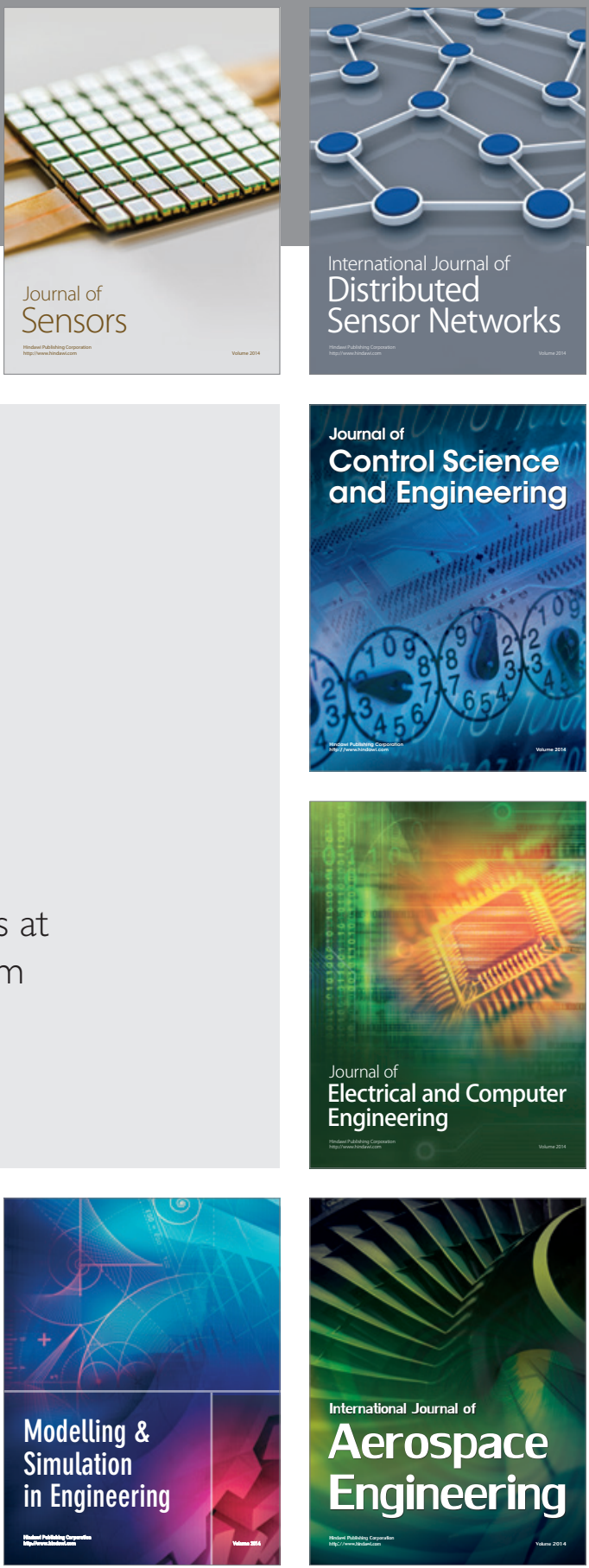

Journal of

Control Science

and Engineering
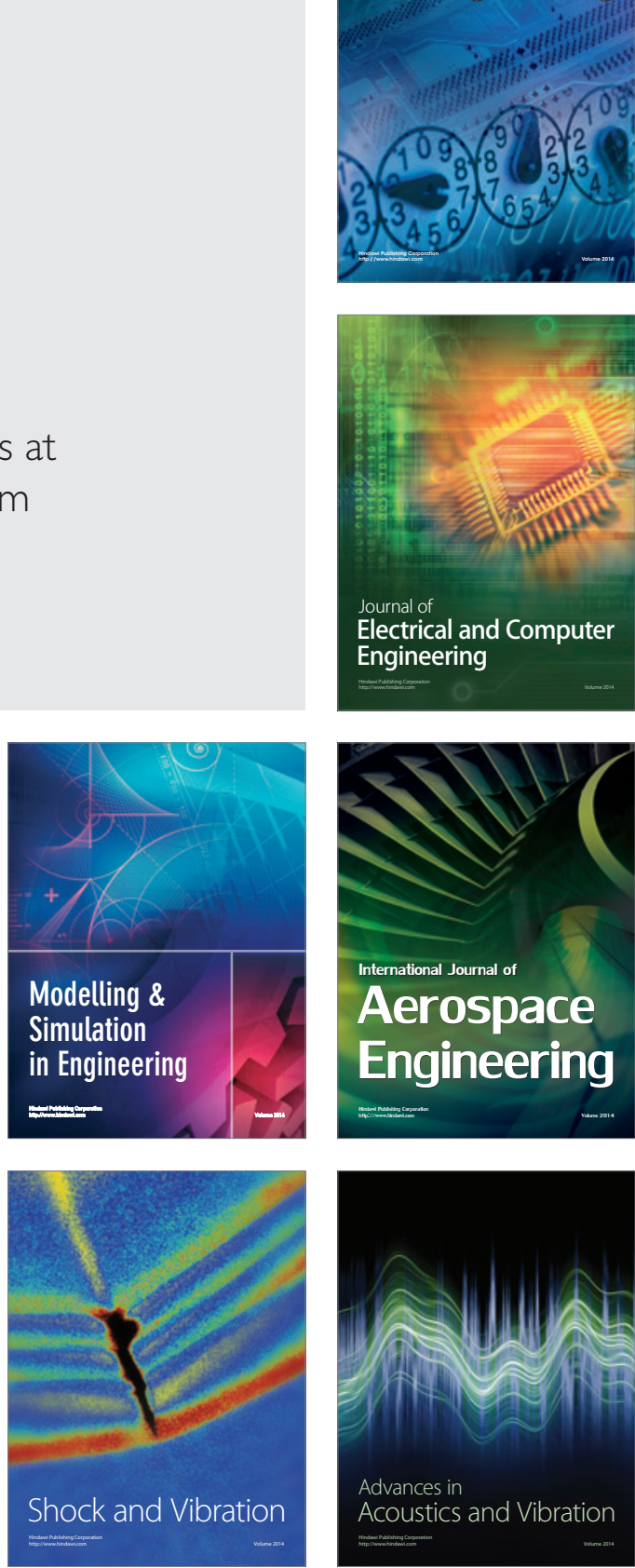